\title{
PsycWord: A Windows program for preparing clinical documentation in psychology
}

\author{
BARRY A. TANNER \\ Wayme State University, Detroit, Michigan
}

\begin{abstract}
PsycWord is designed to guide the user through the process of rapidly producing psychological reports. Modules may be mixed to produce a variety of reports. Windows controls and standardization decrease the time needed for creating clinical reports, while flexibility is built in to encourage customization and the use of professional judgment. The program is most suitable for adult outpatients, but may be useful with other populations as well.
\end{abstract}

Structured computer reporting of technical medical findings has resulted in greater completeness than traditional free-text reports have (Gouveia-Oliveira et al., 1991; Kent, Shortliffe, Carlson, Bischoff, \& Jacobs, 1985; Kuhn et al., 1992). While lists of all possible descriptors provide prompts that increase the completeness of reports, highly structured approaches are likely to be used only if the user believes the costs are outweighed by the benefits (Moorman, van Ginneken, van der Lei, \& van Bemmel, 1994). Tanner has described programs with varying degrees of such flexibility for several specialized areas of psychology, nursing, and psychiatry (Tanner, 1993a, 1993b, 1994; Tanner, Marcolini, Howell, Bateau, \& Faber-Bermudez, 1995). This paper describes a new program, PsycWord, which attempts to maximize both structure and flexibility by (1) presenting lists of terms from which the user can select or insert a descriptor of his/her own choice; (2) using modules that can be mixed to allow many different types of clinical output, for example, combining a history, mental status exam, and treatment plan; and (3) including a built-in word processor, which allows almost unlimited customization of output.

All PsycWord sessions begin and end at the word processor, which allows access to all of the other modules. The user typically begins a report with the Title and Heading data module, identifying the nature of the report, how the subject of the report will be referred to (patient, client, subject, etc.), and whether this is an exam, an evaluation, and so forth. Figure 1 shows the word processor with the Title and Heading module selected from the data menu of the word processor.

Additional modules are selected as appropriate to the report. An initial evaluation might add the Psychosocial, Mental Status, and Summary and Diagnosis modules to the report. Each of these modules is itself organized around on-screen file folders with labels such as Presenting Com-

The author's address is Department of Psychiatry and Behavioral Neurosciences, University Psychiatric Center-Central Campus, Wayne State University, Detroit, MI 48201 (e-mail: btanner@med.wayne.edu). plaint, Prior-Illness, Personal \& Family History, Social Situation, Appearance, Speech, Perception, and Thought Content.

Data are entered by selecting from lists of choices provided in combo boxes, as with "Information obtained from," by clicking on radio buttons for mutually exclusive choices, as in "stated" versus "implied," or by typing in free-form text, as in "Development of psych Symptoms," all in the Presenting Complaint folder shown in Figure 2 .

Some items are context sensitive. For example, if the user indicates that the subject has served in the military, controls are presented for gathering information regarding combat experience, type of discharge, and rank. Otherwise, these controls are invisible. Similarly, controls are presented regarding the impact of prior illness, types of prior treatment, and the impact of prior treatment only if the subject is identified as having had prior episodes of illness. Figure 3 illustrates the Prior Psych Illness Folder with all of the controls visible and with the combo box for "Types of prior Treatment" open in order to allow selecting from it.

The word-processing component allows the user to modify the report produced by the program, save to disk, load the saved report again later, and print hard copies. While not explicitly designed for use with any particular psychiatric population, the program is probably best suited for adult outpatients. Early use within a university outpatient clinic suggests that PsycWord saves user time, helps students in particular to organize their reports, and improves clinical documentation.

PsycWord is written in Visual Basic 4.0 and uses the HighEdit custom control. The installation program includes all necessary files in compressed form on three high-density 3.5-in. disks. Readers interested in obtaining a copy of PsycWord should contact the author for a copy of the necessary form to complete. Upon receipt of the completed form, three formatted high-density disks, and a stamped, self-addressed mailer for the three disks, the author will mail a copy of the program back. 


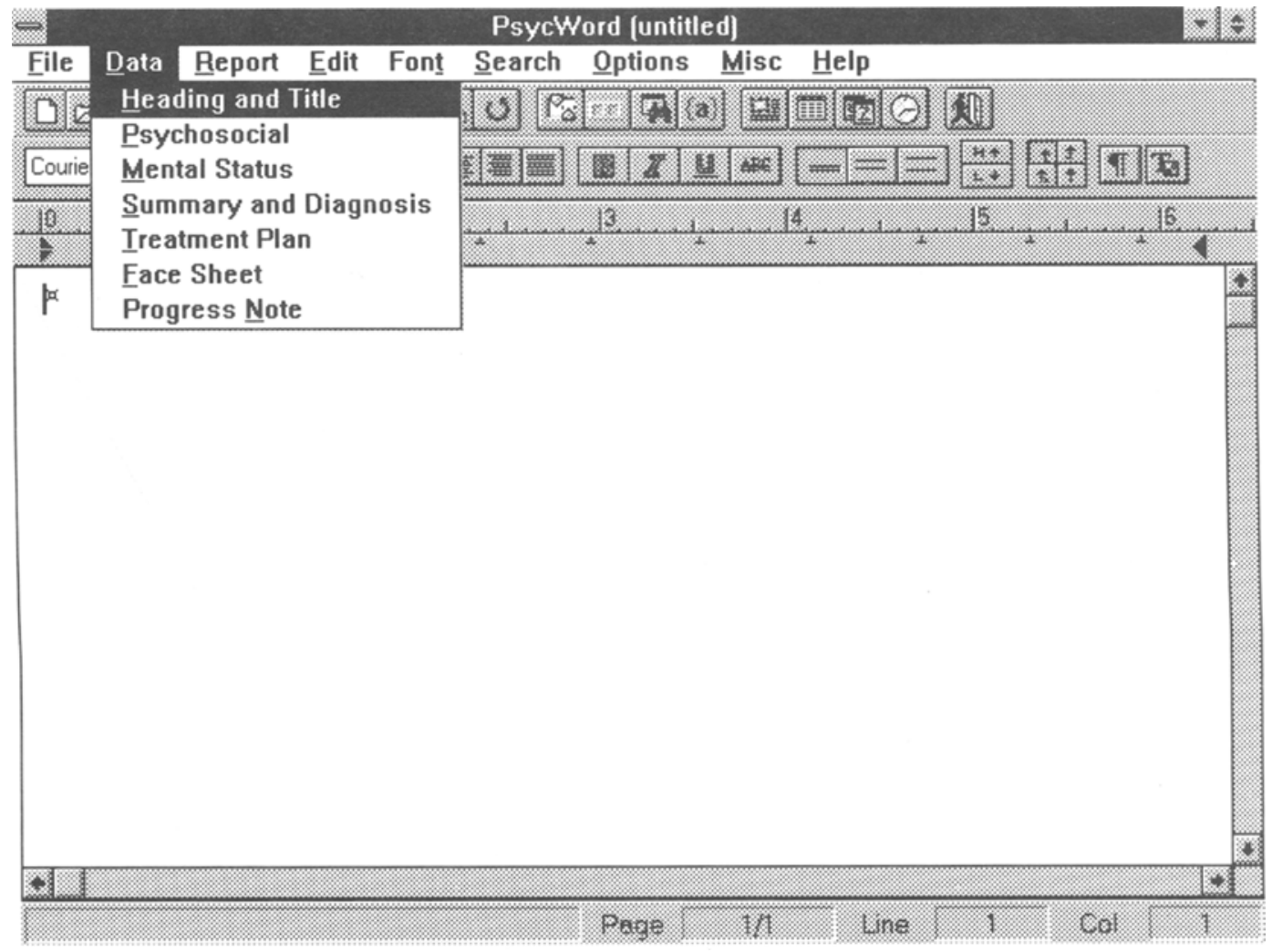

Figure 1. The word processing module with the Heading and Title data module selected.

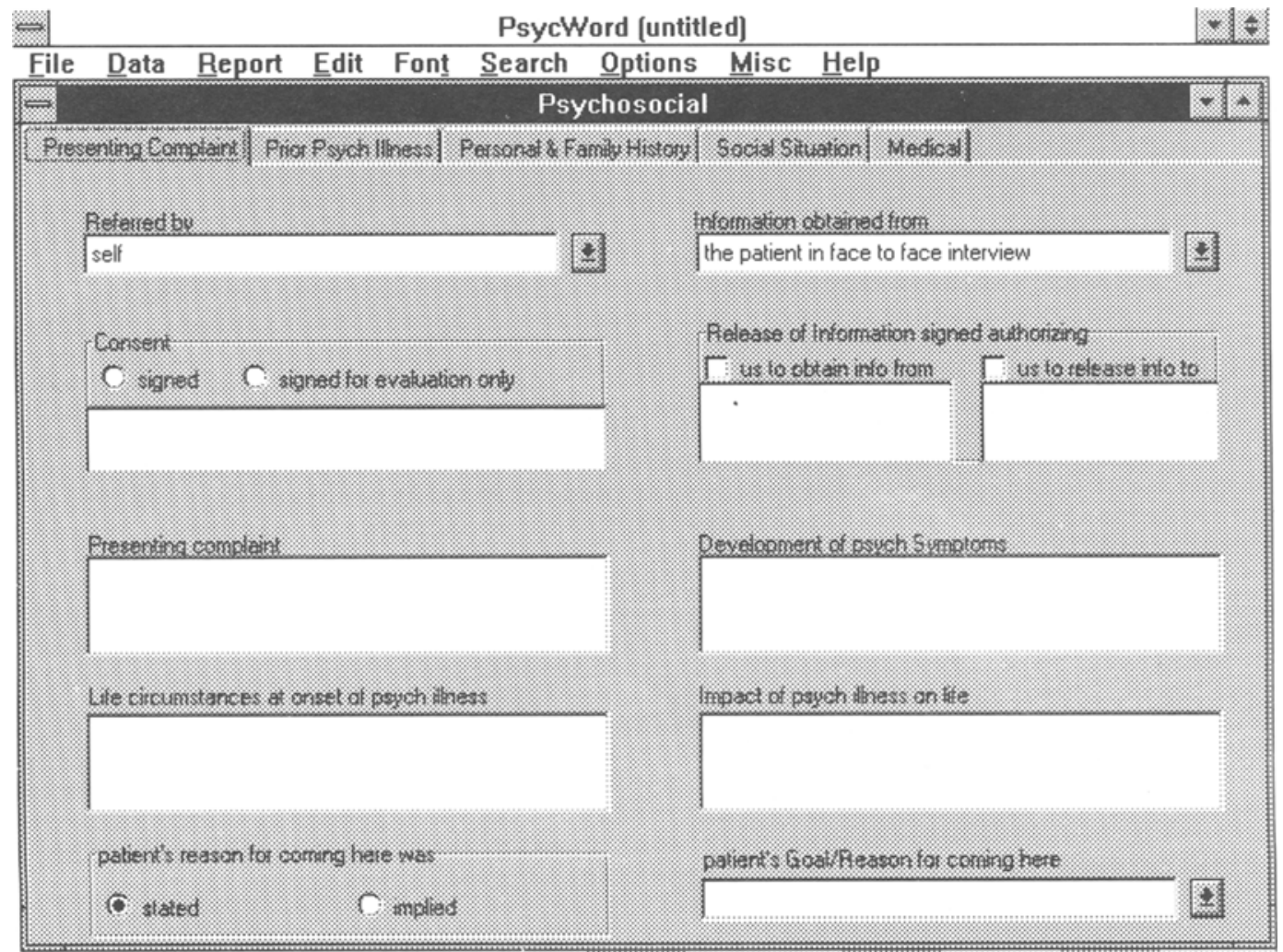

Figure 2. The Psychosocial module open to the Presenting Complaint folder. 


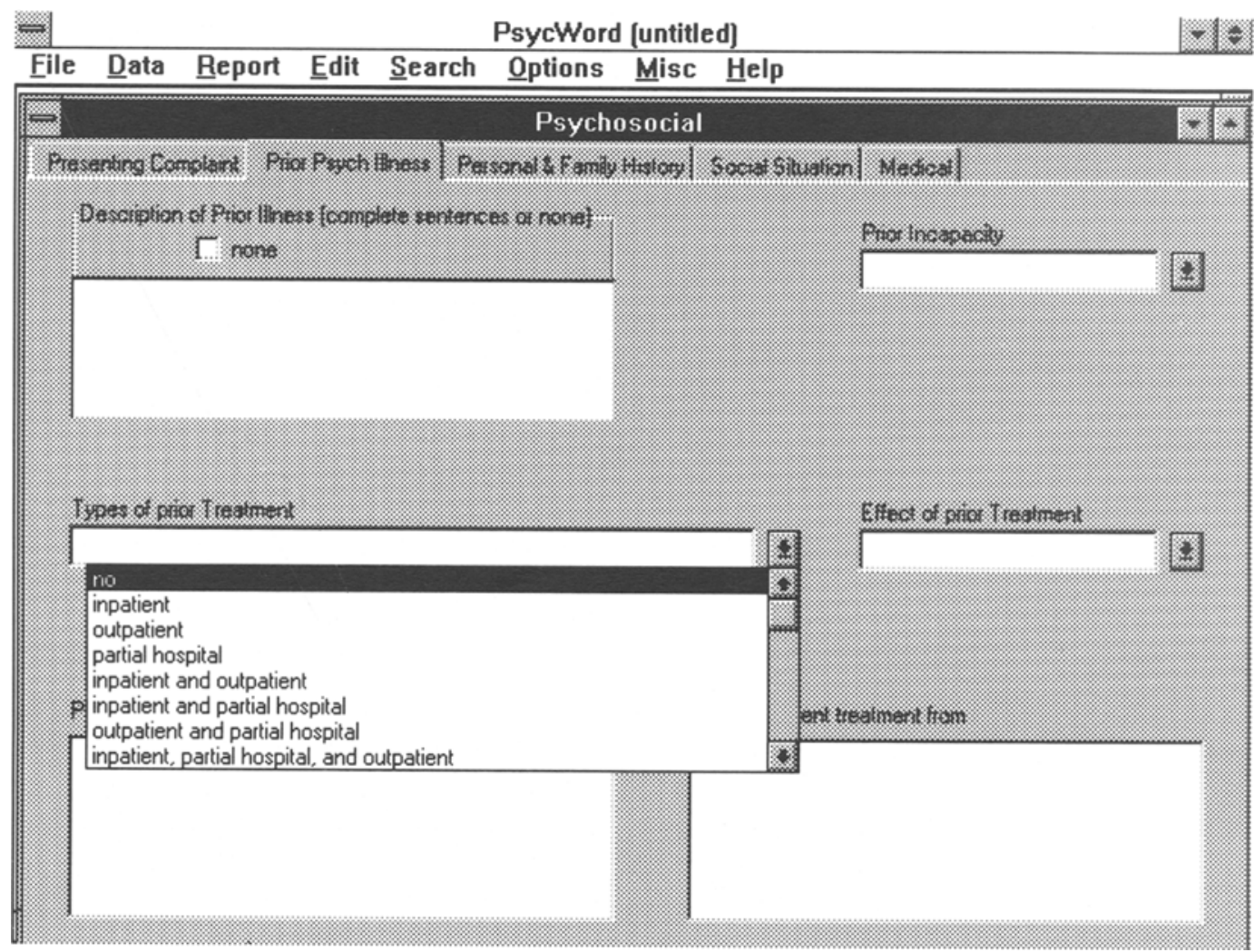

Figure 3. The Prior Psych Illness folder with the Types of prior Treatment combo box open for selection.

\section{REFERENCES}

Gouveia-Oliveira, A., Raposo, V. D., Salgado, N. C., Almeida, I., Nobre-Leitao, C., \& Galvao de Melo, F. (1991). Longitudinal comparative study on the influence of computers on reporting of clinical data. Endoscopy, 23, 334-337.

Kent, D. L., Shortliffe, E. H., Carlson, R. W., Bischoff, M. B., \& $J_{A C O B S}, C . D$. (1985). Improvements in data collection through physician use of a computer-based chemotherapy treatment consultant. Journal of Clinical Oncology, 3, 1409-1417.

Kuhn, K., Gaus, W., Wechsler, J. G., Janowitz, P., Tudyka, J., Kratzer, W., Swobodnik, W., \& Ditschuneit, H. (1992). Structured reporting of medical findings: Evaluation of a system in gastroenterology. Methods of Information in Medicine, 31, 268-274.

Moorman, P. W., van Ginneken, A. M., VAN Der Lei, J., \& van BeMMEL, J. H. (1994). A model for structured data entry based on explicit descriptional knowledge. Methods of Information in Medicine, 33, 454-463.
TANNER, B. A. (1993a). Computer-aided reporting of the results of mental retardation evaluations. Behavior Research Methods, Instruments, \& Computers, 25, 203-207.

TANNER, B. A. (1993b). Computer aided reporting of the results of neuropsychological evaluations of traumatic brain injury. Computers in Human Behavior, 9, 51-56.

TANNER, B. A. (1994). The mental retardation report framework. Behavior Research Methods, Instruments, \& Computers, 26, 213-214.

Tanner, B. A., Marcolini, R. C., Howell, E., Bateau, J., \& FaberBERMUDEZ, I. (1995). A Windows program to assist in preparing reports of emergency psychiatry nursing assessments. Behavior Research Methods, Instruments, \& Computers, 27, 166-168.

(Manuscript received October 22, 1996; revision accepted for publication December $5,1996$. 